\title{
Dynamical characteristics of nano-lasers subject to optical injection and phase conjugate feedback
}

Han, Hong; Shore, K. Alan

\section{IET Optoelectronics}

\author{
DOI: \\ 10.1049/iet-opt.2017.0058
}

Published: 01/02/2018

Peer reviewed version

Cyswllt i'r cyhoeddiad / Link to publication

Dyfyniad o'r fersiwn a gyhoeddwyd / Citation for published version (APA):

Han, H., \& Shore, K. A. (2018). Dynamical characteristics of nano-lasers subject to optical injection and phase conjugate feedback. IET Optoelectronics, 12(1), 25-29. https://doi.org/10.1049/iet-opt.2017.0058

\footnotetext{
Hawliau Cyffredinol / General rights

Copyright and moral rights for the publications made accessible in the public portal are retained by the authors and/or other copyright owners and it is a condition of accessing publications that users recognise and abide by the legal requirements associated with these rights.

- Users may download and print one copy of any publication from the public portal for the purpose of private study or research.

- You may not further distribute the material or use it for any profit-making activity or commercial gain

- You may freely distribute the URL identifying the publication in the public portal ?
}

Take down policy

If you believe that this document breaches copyright please contact us providing details, and we will remove access to the work immediately and investigate your claim. 


\title{
Dynamical Characteristics of Nano-Lasers Subject to Optical Injection and Phase Conjugate Feedback
}

\author{
Hong Han ${ }^{1,2^{*}}$, K. Alan Shore ${ }^{1}$ \\ ${ }^{1}$ School of Electronic Engineering, Bangor University, Wales, LL57 1UT, UK \\ ${ }^{2}$ College of Physics and Optoelectronics, Key Laboratory of Advanced Transducers and Intelligent Control \\ System, Ministry of Education, Taiyuan University of Technology, Taiyuan 030024, China \\ *hanhong@tyut.edu.cn
}

\begin{abstract}
The dynamics of nano-lasers has been analysed using rate equations which include the Purcell cavity-enhanced spontaneous emission factor $F$ and the spontaneous emission coupling factor $\beta$. It is shown that when subject to optical injection and phase conjugate feedback nano-lasers may exhibit remarkably stable small-amplitude oscillations with frequencies of order $300 \mathrm{GHz}$. Critically it is established that such oscillations persist when the effects of noise are taken into account. The appearance of such high-frequency oscillations is associated with the effective reduction of the carrier lifetime for larger values of the Purcell factor, $F$, and spontaneous coupling factor, $\beta$. The effects of the feedback distance and bias currents are also considered. As the optical injection strength increases for fixed phase conjugate feedback and relatively short feedback distances, the nano-laser displays periodic dynamics and then enters stable locking. As the feedback distance increases the quasi-periodic dynamics dominates. Increased bias current can also induce quasi-periodic behaviour albeit this may be ameliorated by reducing the strength of the phase conjugate feedback.
\end{abstract}

\section{Introduction}

The dynamical properties of semiconductor laser subject to external perturbations has been a topic of interest for many decades. Such external perturbations may include optical injection as well as regular and phase conjugate feedback. The prospect of developing nano-lasers with distinctive physical properties has stimulated several analyses of their dynamical behaviour. It is important to appreciate that fabrication of nano-lasers presents a formidable technical challenge. Despite effort over a decade or so [see e.g. 1-12] relatively few examples of such devices being reported. Those efforts have been accompanied by pioneering work on a theoretical description of nano-laser dynamics [13].

However, as far as the present authors are aware, no experimental results on the dynamics of particular nano-laser designs have been published. In that context it is appropriate to undertake theoretical explorations of nano-laser dynamical behaviour using a generic model for nano-lasers. Particular attention is given to the effects on nano-lasers of Purcellenhanced spontaneous emission and/or relatively large values of the spontaneous emission coupling factor Work by Gu. et al. [14] and Gerard et al. [15] has included detailed calculation of the spontaneous emission rate in nano-lasers. This work has shown that there is an interdependence between the spontaneous emission coupling factor and the Purcell enhancement factor. However, the precise relationship between these two factors is dependent upon the specific nano-laser structure under consideration and thus are | not suitable for generic modelling on nano-laser dynamics as being undertaken here. Thus, whilst being cognisant of [14] and [15], in the present work the Purcell factor and the spontaneous emission factor are taken to be independent parameters. In this way it is possible to identify the trends in device performance consequent to changes in these two parameters. It is fully recognised, however, that in a practical context there will be constraints on the accessible values of these parameters and thus not all combinations of values of these parameters treated here will necessarily be available.

Using such an approach exploration has been undertaken of a number of aspects of nano-laser dynamics [6 21] including the impact of optical injection [19] conventional [17 ] and phase conjugate [18] optical feedback. A salient feature of that analysis is that nano-laser appear to hold the prospect of being relatively robust to external perturbations. Recent work has also investigated the control of instabilities in nano-lasers [22].

Optical injection is well-known as a means for enhancing the modulation bandwidth of semiconductor laser [23] and in modulation bandwidth enhancement in mutuallycoupled monolithically integrated laser diodes has been reported [24].

Attention has been given to the dynamical behaviour of mutually-coupled nano-lasers $[20,21]$ including an exploration of effects of modulation in mutually coupled nano-lasers [21]. Those studies identified regimes of operation where the coupled nano-lasers may be individually modulated without affecting each other - so-called zerocross-talk regimes. The existence of such regimes reinforces the view that nano-lasers operation is often immune to external perturbations.

In the present work the behaviour of nano-lasers when subject to both optical injection and phase conjugate feedback. In addition attention is given to the effects of feedback distancenoise on the predicted dynamical Commented [h1]: In this paper, we didn't add noise. Shall we behaviour. The application of phase conjugate feedback to change it as feedback distance? conventional semiconductor lasers has been widely studied both theoretically and experimentally [25-40] but, to date only one study has been made of phase conjugate effects on nano-lasers [18]. The combination of phase conjugate 
feedback and optical injection offers opportunities for the novel dynamical responses discussed in the present work.

\section{Nano-laser dynamics}

A schematic diagram of nano-laser under optical injection and optical feedback is shown in Fig. 1. This system is modelled using modified forms of rate equations which incorporate the Purcell enhanced spontaneous emission factor, $\mathrm{F}$ and spontaneous emission coupling factor, $\beta$ have been included as introduced in [8].

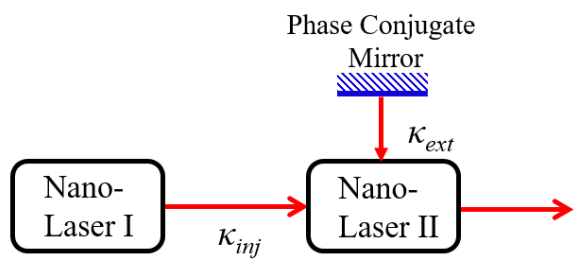

Fig. 1. Schematic diagram semiconductor nano-lasers with optical injection and external phase conjugate mirror feedback.

$$
\begin{aligned}
\frac{d S_{\mathrm{I}}(t)}{d t}= & \Gamma\left[\frac{F \beta N_{\mathrm{I}}(t)}{\tau_{n}}+G_{n}\left(N_{\mathrm{I}}(t)-N_{t h}\right) S_{\mathrm{I}}(t)\right] \\
\frac{d \phi_{\mathrm{I}}(t)}{d t}= & \frac{\alpha}{2} \Gamma G_{n}\left(N_{\mathrm{I}}(t)-N_{t h}\right) \\
\frac{d S_{\mathrm{II}}(t)}{d t}= & \Gamma\left[\frac{F \beta N_{\mathrm{II}}(t)}{\tau_{n}}+G_{n}\left(N_{\mathrm{II}}(t)-N_{t h}\right) S_{\mathrm{II}}(t)\right] \\
+ & \frac{2 \kappa_{i n j}}{\tau_{\text {in }}} \sqrt{S_{\mathrm{II}}(t) S_{\mathrm{I}}(t)} \cos \Theta_{i n j}(t) \\
+ & \frac{2 \kappa_{e x t}}{\tau_{i n}} \sqrt{S_{\mathrm{II}}(t) S_{\mathrm{II}}\left(t-\tau_{e x t}\right)} \cos \Theta_{e x t}(t) \\
\frac{d \phi_{\mathrm{II}}(t)}{d t}= & \frac{\alpha}{2} \Gamma G_{n}\left(N_{\mathrm{II}}(t)-N_{t h}\right)-2 \pi \Delta f \\
& -\frac{\kappa_{i n j}}{\tau_{i n}} \sqrt{\frac{S_{\mathrm{I}}(t)}{S_{\mathrm{II}}(t)}} \sin \Theta_{i n j}(t) \\
& -\frac{\kappa_{e x t}}{\tau_{i n}} \sqrt{\frac{S_{\mathrm{II}}\left(t-\tau_{e x t}\right)}{S_{\mathrm{II}}(t)}} \sin \Theta_{e x t}(t) \\
\frac{d N_{\mathrm{II}}(t)}{d t}= & \frac{I_{\mathrm{II}}}{e V_{b}}-\frac{N_{\mathrm{II}}(t)}{\tau_{n}}(F \beta+(1-\beta)) \\
& -G_{n}\left(N_{\mathrm{II}}(t)-N_{0}\right) S_{\mathrm{II}}(t) \\
\Theta_{i n j}(t)= & -2 \pi \Delta f t+\phi_{\mathrm{II}}(t) \\
\Theta_{e x t}(t)= & \phi_{\mathrm{II}}(t)+\phi_{\mathrm{II}}\left(t-\tau_{e x t}\right)
\end{aligned}
$$

It is underlined that the Purcell factor and the spontaneous emission coupling factor impact the spontaneous emission rate as shown in Eqs. (1)- (4) below, wherein Eqs. (1) and (2) are for the driving laser (laser I), and Eqs. (3) and (4) are for the response laser (laser II) which is concerned in this paper. Specifically it is pointed out that for Purcell factors greater than unity an effective reduction in the carrier lifetime will result. Similarly an increase of the spontaneous emission coupling factor towards unity also causes an effective reduction of the carrier lifetime. Such a reduction is seen to be pertinent to the emergence of high-frequency oscillations discussed in the present paper. In contrast, the phase Eq. (5) is dependent on the laser gain and hence is not affected by the enhanced spontaneous emission.

In the rate equations the subscripts 'I' and 'II' represent laser I and laser II respectively. $S(t)$ is the photon density and $N(t)$ is the carrier density, $\varnothing(t)$ is the phase of laser, $\Theta(t)$ is the phase of laser II, where 'inj' and 'ext' represent injection and external feedback. $\Gamma$ is the confinement factor; $\tau_{n}$ and $\tau_{p}$ are the radiative carrier lifetime and photon lifetime respectively. $G_{n}$ is the differential gain that takes into account the effect of group velocity, $N_{o}$ is the transparency carrier density, $\epsilon$ is the gain saturation factor and $\alpha$ is the linewidth enhancement factor. $I=j I_{t h}$ is the dc bias current, where $j$ is the normalized injection current $; I_{t h}$ is the threshold current $\left(I_{t h}=(F \beta+(1-\beta)) N_{t h} e V_{a} / \tau_{n}\right), \quad V_{a}$ is the volume of the active region, $e$ is the electron charge and $N_{t h}\left(N_{t h}=N_{o}+1 / \Gamma g_{n} \tau_{p}\right)$ is the threshold carrier density. $\Delta f=f_{\mathrm{I}^{-}}$

\begin{tabular}{|c|c|c|c|}
\hline Wavelength & $\lambda$ & $1591 \mathrm{~nm}$ & [12] \\
\hline Cavity length & $L$ & $1.39 \mu \mathrm{s}$ & [12] \\
\hline Volume of active region & $V_{a}$ & $3.96 \times 10^{-19} \mathrm{~m}^{3}$ & [12] \\
\hline Group refractive index & $n$ & 3.4 & [12] \\
\hline $\begin{array}{l}\text { Round-trip time in inner } \\
\text { cavity }\end{array}$ & $\tau_{\text {in }}$ & $0.032 \mu \mathrm{s}$ & [12] \\
\hline Photon lifetime & $\tau_{\mathrm{p}}$ & $0.36 \mathrm{ps}$ & [12] \\
\hline Carrier lifetime & $\tau_{\mathrm{n}}$ & $1 \mathrm{~ns}$ & [41] \\
\hline Differential gain & $G_{\mathrm{n}}$ & $1.65 \times 10^{-12} \mathrm{~m}^{-3} / \mathrm{s}$ & [12] \\
\hline Mode confinement factor & $\Gamma$ & 0.645 & [12] \\
\hline $\begin{array}{l}\text { Line-width enhancement } \\
\text { factor }\end{array}$ & $\alpha$ & 5 & [42] \\
\hline $\begin{array}{l}\text { Transparency carrier } \\
\text { density }\end{array}$ & $N_{0}$ & $1.1 \times 10^{24} \mathrm{~m}^{-3}$ & [41] \\
\hline $\begin{array}{l}\text { Laser facet power } \\
\text { reflectivity }\end{array}$ & $R$ & 0.85 (estimated) & \\
\hline Injection power ratio & $R_{i n j}$ & $0-1$ & \\
\hline External power reflectivity & $R_{\text {ext }}$ & $0-0.01$ & \\
\hline $\begin{array}{l}\text { Normalized injection } \\
\text { current }\end{array}$ & $j$ & $2-4$ & \\
\hline Feedback delay/distance & $\tau_{\mathrm{ext}} / D_{e x t}$ & $0.03 \mathrm{~ns} / 0.005 \mathrm{~m}$ & \\
\hline Cavity Purcell factor & F & 10 & \\
\hline $\begin{array}{l}\text { Spontaneous emission } \\
\text { coupling }\end{array}$ & $\beta$ & 0.1 & \\
\hline
\end{tabular}
$f_{\mathrm{II}}$ is the frequency detuning between laser I, $f_{\mathrm{I}}$, and laser II, $f_{\text {II. }} \tau_{e x t}=2 D_{\text {ext }} / c$ is the feedback delay, where $D_{\text {ext }}$ is the distance of the external-cavity. $\tau_{\text {in }}=2 n L / c$ is the round-trip time in of the laser cavity, where $L$ is the cavity length and $n$ is group refractive index. The optical injection into the laser II is controlled by the injection fraction, $\kappa_{i n j}$.

$$
\kappa_{i n j}=\frac{(1-R) \sqrt{R_{i n j}}}{\sqrt{R}}
$$

Where, $R_{i n j}$ is the rate of the injected electric field power, $R$ is the reflectivity of the laser.

Table 1 Nano-laser device parameters 
The external-cavity feedback strength into laser II is controlled by feedback fraction, $\kappa_{\text {ext }}$.

$$
\kappa_{e x t}=\frac{(1-R) \sqrt{R_{e x t}}}{\sqrt{R}}
$$

Where, $R_{e x t}$ is the feedback power ratio including coupling losses. The dynamics of the nanolasers is analysed using the device parameters given in Table I.

Attention is drawn to the fact that an increase of spontaneous emission via the Purcell factor, $F$ or the spontaneous emission coupling factor $\beta$ may lead to a change in the laser threshold current [21]. This has been taken into account in the present analysis.

\section{Results}

The aim of this paper is to explore the dynamical behaviour of nano-lasers subject to both optical injection and phase conjugate feedback.

Firstly, we show the bifurcation diagram for the case of optical injection into nano-laser but with a fixed strength of phase conjugate feedback - taken to be $-25 \mathrm{~dB}$. The results are shown in Fig. 2 where $F=10, \beta=0.1$ and the laser is biased at twice the threshold current. The parameters of the master laser and response laser are assumed to be identical. In this case, the injection photon density is fixed at $2.64 \times$ $10^{21} \mathrm{~m}^{-3}$, that is the output of the master nano-laser at $2 I_{t h}$. Fig.2 shows that the nano-laser initially exhibits an oscillatory behaviour (period-1, i.e. corresponding to two points) and then undergoes period-doubling (period-2 i.e. corresponding to two points) before returning to period-1 again, then enter a regime of stable locking (i.e. corresponding to one point) finally as the optical injection increases.

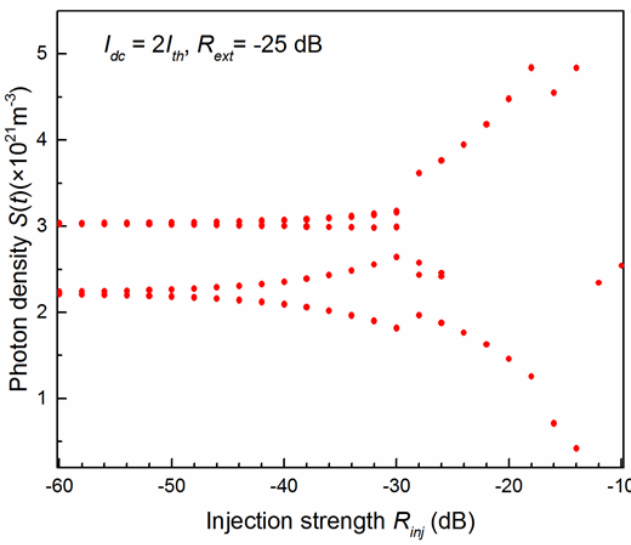

Fig.2. Bifurcation diagram of response laser vs. optical injection with $-25 d B$ phase conjugate feedback for $F=10$, $\beta=0.1, I_{d c}=2 I_{t h}, D=0.5 \mathrm{~cm}$

The corresponding dynamics of the nanolaser is shown in Figs. 3(a) to 3(d) as the injection couplings are fixed at $-60 \mathrm{~dB},-40 \mathrm{~dB},-20 \mathrm{~dB}$ and $-12 \mathrm{~dB}$ respectively. The amplitude of period-1 oscillations (Fig.3(a)) and period-2 oscillations (Fig. 3(b)) are shown to be basically the same as may be deduced from Fig. 2. For the initial period-1 behaviour, FFT of photon density time series is shown in Fig. $3(\mathrm{e})$, the oscillation frequency is of order $600 \mathrm{GHz}$. The appearance of such high-frequency oscillations being associated with the effective reduction of the carrier lifetime for larger values of the Purcell factor, $F$, and spontaneous coupling factor, $\beta$

When, with increasing optical injection, the lase returns to period-1 behaviour (Fig. 3(c)), the oscillation amplitude is significantly increased ( as expected from Figure 2), however the oscillation frequency (Fig. 3(f)) is about half that in the Fig.3(a) case. As the optical injection strength increases further, the nano-laser enters into the stable locking state as shown in Fig. 3(d).
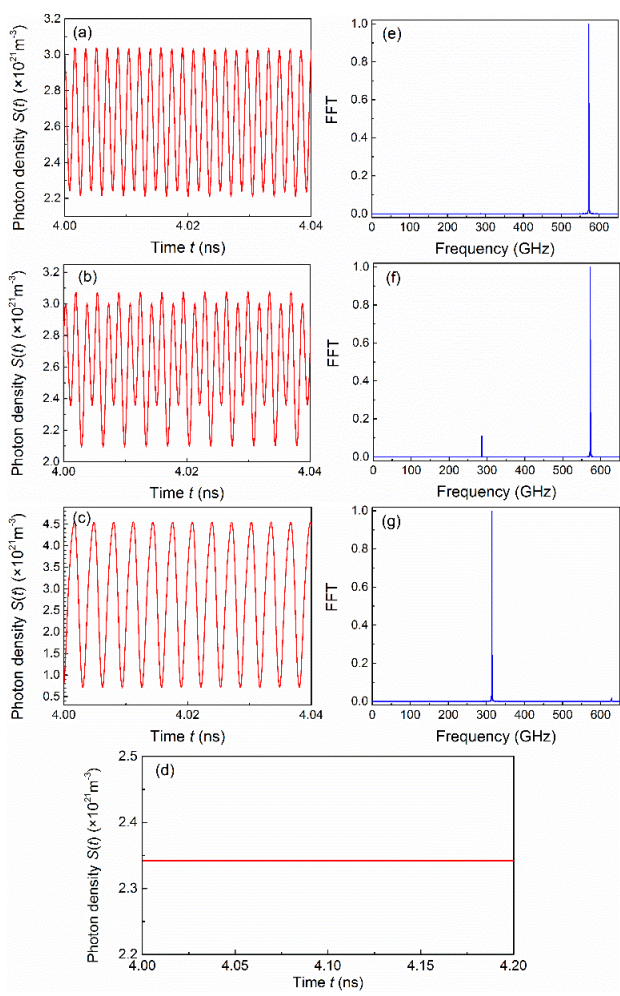

Fig. 3. Time series of photon density of response nano-lase at $R_{\text {ext }}=-25 \mathrm{~dB}:\left(\right.$ a) period -1 at $R_{\text {inj }}=-60 \mathrm{~dB} ;(\boldsymbol{b})$ period -2 at $R_{i n j}=-40 \mathrm{~dB} ;(\boldsymbol{c})$ period -1 at $R_{\text {inj }}=-16 \mathrm{~dB} ;(\boldsymbol{d})$ stable locking at $R_{i n j}=-12 \mathrm{~dB}$. The corresponding oscillation frequency for $(\boldsymbol{a})$ (c) are shown in $(\boldsymbol{d})-(f)$, respectively.

To portray more clearly the impact of the distance to the phase conjugate mirror, calculations have been performed of the various dynamical regions which appear as the as distance changes. The results are shown in Fig. 6. It is seen that as the distance increases the quasi-periodic region replaces the period-1 oscillations found at shorter distances and shown with black squares. In addition, the period-2 region significantly shrinks. With increase of the feedback distance to a relatively large value of $1 \mathrm{~cm}$, the response nano- 
laser undergoes quasi-periodic to period-1 and then enters stable locking. Further increase of the feedback distance results in the period-1 region being further decreased.

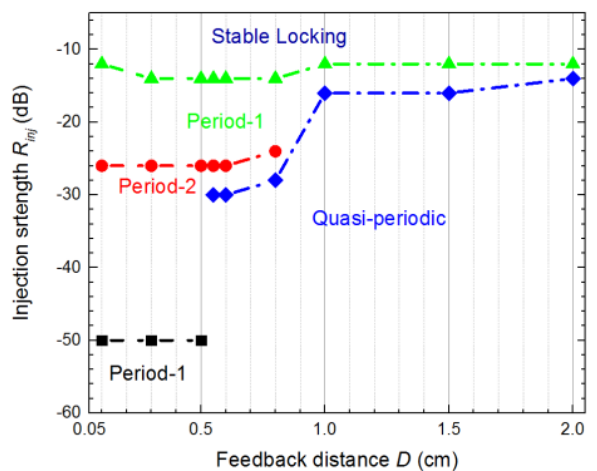

Fig.6. Dynamical regions of response laser vs. feedback distance. The black squares, the red circles, the green triple denote the threshold of period-2, period-1, and the stable locking. The interval regions are the initial period-1, period2 , period-1 from down to up. The blue diamond and its below present quasi-periodic region. The interval between blue diamond and red circles is the period-2 region, the interval between blue diamond and green triple is the period-1 region.

Figure 7 shows that if the laser bias currents are increased to $4 I_{t h}$, (with the input photon density increasing to $5.8 \times 10^{21} \mathrm{~m}^{-3}$ ) the response laser become unstable. Here quasi-periodic behaviour appears instead of the stable period1 and period- 2 oscillations found at the lower bias currents. The laser proceeds from the quasi-periodic regime to the stable-locking state. This shows that in order to obtain a stable periodic output state or stable locking, the bias current should not be too large.

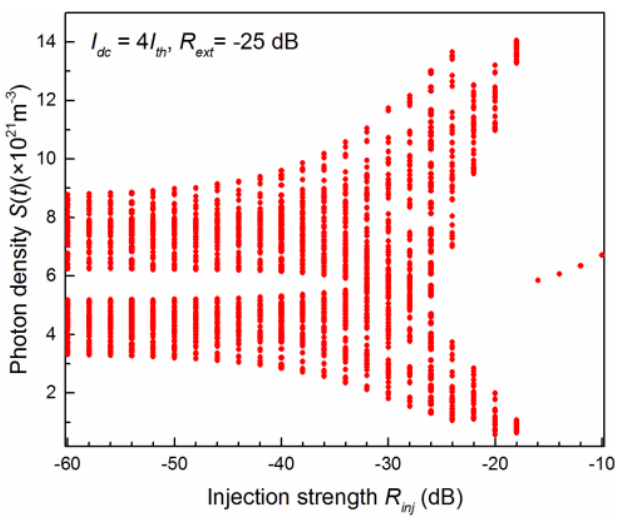

Fig.7. Bifurcation diagram of response laser vs. optical injection with $-25 d B$ phase conjugate feedback at $F=10$, $\beta=0.1, I_{d c}=4 I_{t h}, D=0.5 \mathrm{~cm}$
Alternatively, stable periodic outputs can be obtained for weaker phase conjugate feedback. Relevant results are shown in Fig. 8, where the phase conjugate feedback strength is decreased to $-30 \mathrm{~dB}$. In this case, stable periodic oscillations are recovered and the laser transitions from periodic dynamics to stable locking as the optical injection strength increases.

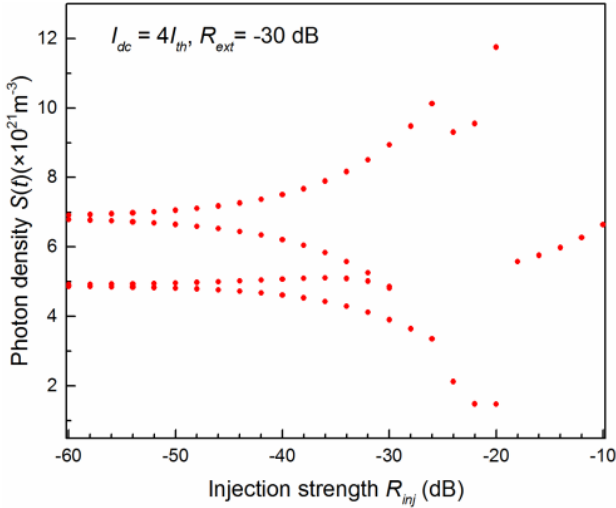

Fig.8. Bifurcation diagram of response laser vs. optical injection with $-30 \mathrm{~dB}$ phase conjugate feedback at $F=10$, $\beta=0.1, I_{d c}=4 I_{t h}, D=0.5 \mathrm{~cm}$.

\section{Conclusions}

Theoretical analysis undertaken in this work shows that both driving current and feedback distance in optical injection nano-laser with phase conjugate feedback can significantly affect the dynamics of the response nano-laser. Apart from the phase conjugate feedback strength, increasing the bias current and feedback distance can induce instabilities in the form of quasi-periodic oscillation. For relatively short feedback distance, a high oscillation frequency in order $300 \mathrm{GHz}$ is observed. The observed behaviour offers interesting opportunities for generating stable high-frequency oscillations using nano-lasers with optical injection and phase conjugate feedback including, for example, for use as clock pulses in photonic integrated circuits.

\section{Acknowledgments}

This work is supported by the Sêr Cymru National Research Network in Advanced Engineering and Materials, the International Science \& Technology Cooperation Program of China (2014DFA50870), the National Natural Science Foundation of China (61527819, 61475111, 61601319), the Natural Science Foundation of Shanxi Province(201601D202043), and the Outstanding Innovative Teams of Higher Learning Institutions of Shanxi.

\section{References}

[1] Pelton, M., Vuckoviá, J., Solomon, G., et al.: 'Efficient source of a single photons: a single quantum dot in a micropost microcavity ', Phys. E, 2003, 17, pp 564-567 
2] Reitzensteinm, S., Heindel, T., Kistner, C., et al.: 'Low threshold electrically pumped quantum dot-micropillar lasers', Appl. Phys. Letts., 2008, 93, 6, pp 061104-1 061104-3

4] Hill, M. T., Marell, M., Leong, E. S. P.,et al.: 'Lasing in metal-insulator-metal sub- wavelength plasmonics waveguides', Opt. Exp., 2009, 17,13, pp. 11107-11112 [5] Oulton, R. F., et al.: 'Plasmon lasers at deep subwavelength scale', Nature, 2009, 461, pp. 629-632

[6] Yu, K., Lakhani, A., Wu, M. C., 'Subwavelength metaloptic semiconductor nanopatch lasers', Opt. Express., 2010, 18, 9, pp. 8790-8799

[7] Ning, C. Z.: 'Semiconductor nanolasers', Phys. Status. Solidi. B, 2010, 247, pp 774

[8] Ding, K., Liu, Z., Yin, L., et al.: 'Electrical injection, continuous wave operation of sub wavelength-metallic-cavity lasers at 260 K', Appl. Phys. Lett., 2011, 98, 23, pp 231108 $1-231108-3$

[9] Chuang, S. L., Bimberg, D.: 'Metal-cavity nanolasers', IEEE Photon. J, 2011, 3, 288

[10] Ding, K., Ning, C. Z.: 'Metallic sub-wavelength-cavity semiconductor nanolasers', Light: Sci. Appl., 2012, 1, 7, pp 20

[11] Gao, H., Fu, A., Andrews S. C., et al.: 'Cleaved-coupled nanowire lasers', Proc. Natl. Acad. Sci., 2013, 110, 3, pp 865-869

[12] Ding, K., Hill, M. T., Liu, Z. C., et al.: 'Record performance of electrical injection subwavelength metalliccavity semiconductor lasers at room temperature', Opt. Exp., 2013, 21, 4, pp 4728-4733

13] Suhr, T., Gregerson, N., Yvind Y., et al.: 'Modulation response of nanoLEDs and nanolasers exploiting Purcell enhanced spontaneous emission', Opt. Exp., 2010, 18, 11, pp 11230-11241

[14] Gu, Q., Slutsky, B., Vallini, F., et al.: 'Purcell effect in sub-wavelength semiconductor lasers', Opt. Express., 2013, 21, 13, pp 15603-15617

[15] Gerard, J. M., Gayral, B., et al.: 'InAs quantum dots: artificial atoms for solid-state cavity-quantum electrodynamics', Physica E, 2001, 9, 1, pp 131-139 [16] Sattar, Z. A., Shore, K. A.: 'Analysis of the direct modulation response of nanowire lasers', IEEE. J. Lightw. Technol., 2015, 33, 14, pp 3028 - 3033

[17] Sattar, Z. A., Shore, K. A.: 'External optical feedback effects in semiconductor nano-lasers', IEEE J. Sel. Top.

Quantum Electron., 2015, 21, 6, pp 1800106

[18] Sattar, Z. A., Shore, K. A.: 'Phase conjugate feedback effects on nano-lasers', IEEE J. Quant. Electron., 2016, 52, 4, pp 1100108

[19] Sattar, Z. A., Shore, K. A.: 'Optical Injection Effects in Nano-lasers', IEEE J. Quant. Electron., 2016, 52, 2, pp 1200108

20] Han H., Shore, K. A.: 'Dynamics and stability of mutually coupled nano-lasers', IEEE J. Quant. Electron., 2016, 52, 11, pp 2000306

[21] Han H., Shore, K. A.: 'Modulated mutually coupled nano-lasers', IEEE J. Quant. Electron., 2017, 53, 2, 2000208 [22] Kumar, P., Grillot, F.: 'Control of dynamical instability in semiconductor quantum nanostructures diode lasers: role of phase-amplitude coupling', Eur. Phys. J. Special Topics, 2013, 222, 3-4, pp 813-820

[23] Lau, E. K., Wong L. H., Wu, M. C.: 'Enhanced modulation characteristics of optical injection-locked lasers:
A tutorial', IEEE J. Sel. Top. Quantum Electron., 2009, 15, 3 , pp 618-633

[24] Sun, C., Liu, D., Xiong, B., et al.: 'Modulation charecteristics enhancement of monolithically integrated laser diodes under mutual injection locking', IEEE J. Sel. Top. Quant. Electron., 2015, 21, 6, pp 1802008

[25] Virte, M., Karsaklian Dal Bosco, A., D., et al.: 'Chaos crisis and bistability of self-pulsing dynamics in a laser diode with phase-conjugate feedback', Physical Review A, 2011, 84, pp 043836

[26] Agrawal, G. P., Gray, G. R.: 'Effect of phase-conjugate feedback on the noise characteristics of semiconductor lasers', Phys. Rev. A, 1992, 46, pp 5890-5898

[27] Van Tartwijk, G. H. M., Van Der Linden, H. J. C. Lenstra, D.: 'Theory of a diode laser with phase conjugate feedback', Optics Lett., 1992, 17, pp 1590-1592

[28] Langley, L. N., Shore, K. A.: 'The effect of phase conjugate optical feedback on the intensity noise in laser diode', Optics Lett., 1993, 8, pp 1432-1434

[29] MrCarthy, N., Gay, D.: 'Noise reduction in an argon lase with a phase conjugating external cavity', Optics Lett., 1991, 16, pp 1004-1006

[30] Langley, L. N., Shore, K. A.: 'Intensity noise and linewidth characteristics of laser diodes with phase conjugate optical feedback', IEE. Proc. Optoelectron., 1994, 141, 2, pp $103-108$

[31] Vahala, K., Kyuma, K., Yariv, A., et al.: 'Narrow linewidth single frequency semiconductor laser with a phase conjugate external cavity mirror', Appl. Phys. Lett., 1986, 49, pp 1563-1565

[32] Ohtsu, M. Koshiishi, I., Teramachi, Y.: 'A semiconductor laser as a stable phase conjugate mirror for linewidth reduction of another semiconductor laser', Jap. J Appl. Phys., 1990, 29, L2060-L2062

[33] Agrawal, G. P., Klaus, J. T.: 'Effect of phase-conjugate feedback on semiconductor laser dynamics', Optics Lett. 1991, 16, pp 1325-1327

[34] Andersen, O. K., Fischer, A. P. A., Lane, I. C., et al.. 'Experimental stability diagram of a diode laser subject to weak phase-conjugate feedback from a rubidium vapor cell', IEEE J. Quantum Electron., 1999, 35, 4, pp 577

[35] Sciamanna, M., Shore, K. A.: 'Physics and applications of laser diode chaos', Nature Photonics., 2015, 9, pp 151 162

[36] Lawrence, J. S., Kane, D. M.: 'Contrasting conventional optical and phase-conjugate feedback in laser diodes', Phys. Rev. A., 2001, 63, pp 033805-1 - 033805-10

[37] A Karsaklian Dal Bosco, A., Wolfersberger, D. Sciamanna, M.: 'Extreme events in time-delayed nonlinear optics', Optics Lett., 2013, 38, 5, pp 703 - 705

[38] Mercier, É., Wolfersberger, D., Sciamanna, M. 'Bifurcation to chaotic low-frequency fluctuations in a laser diode with phase-conjugate feedback', Optics Lett., 2014, 39 , 13, pp $4021-4024$

[39] Karsaklian, A., Bosco, D., Wolfersberger, D., et al. 'Super-harmonic self-pulsations from a time-delayed phaseconjugate optical system', Appl. Phys. Lett., 2014, 105, pp 081101-1 - 081101-4

[40] Weicker, L., Erneux, T., Wolfersberger D., et al.: 'Laser diode nonlinear dynamics from a filtered phase-conjugate optical feedback', Phys. Rev., 2015, 92, 2, pp 022906-1 022906 
41] Coldren, L. A., Corzine, S. W., 'Diode Lasers and photonic integrated circuits'. New York, NY, USA: Wiley, 1995

[42] Chang, S. W.: 'Dressed linewidth enhancement factor in small semiconductor lasers', Opt. Express, 2012, 20, 15, pp 16450-16470 